\title{
Inactivation of tankyrases reduces experimental fibrosis by inhibiting canonical Wnt signalling
}

\author{
Alfiya Distler, ${ }^{1}$ Lisa Deloch, ${ }^{1}$ Jingang Huang, ${ }^{1}$ Clara Dees, ${ }^{1}$ Neng-Yu Lin, ${ }^{1}$ \\ Katrin Palumbo-Zerr, ${ }^{1}$ Christian Beyer, ${ }^{1}$ Alexander Weidemann, ${ }^{2}$ Oliver Distler, ${ }^{3}$ \\ Georg Schett, ${ }^{1}$ Jörg H W Distler ${ }^{1}$
}

\begin{abstract}
Handling editor Tore K Kvien
- Additional supplementary data are published online only. To view this file please visit the journal online (http://dx.doi. org/10.1136/annrheumdis2012-202275).

${ }^{1}$ Department of Internal Medicine 3 and Institute for Clinical Immunology, University of Erlangen-Nuremberg, Erlangen, Germany

${ }^{2}$ Department of Internal Medicine 4, Nephrology and Hypertension, University of Erlangen-Nuremberg, Erlangen, Germany

${ }^{3}$ Department of Rheumatology and Center of Experimental

Rheumatology, University Hospital Zurich, Zurich Center of Integrative Human Physiology (ZIHP), Zurich, Switzerland
\end{abstract}

\section{Correspondence to} Dr Jörg H W Distler, Department of Internal Medicine 3 and Institute for Clinical Immunology, University of Erlangen-Nuremberg, Ulmenweg 18, Erlangen 91054, Germany; joerg.distler@uk-erlangen.de

Accepted 21 October 2012 Published Online First 12 November 2012

\section{SLinked}

- http://dx.doi.org/10.1136/ annrheumdis-2012-202450

- http://dx.doi.org/10.1136/ annrheumdis-2012-202775

- http://dx.doi.org/10.1136/ annrheumdis-2012-202864

- http://dx.doi.org/10.1136/ annrheumdis-2013-203261

\section{ABSTRACT}

Objectives Canonical Wnt signalling has recently emerged as a key mediator of fibroblast activation and tissue fibrosis in systemic sclerosis. Here, we investigated tankyrases as novel molecular targets for inhibition of canonical Wnt signalling in fibrotic diseases. Methods The antifibrotic effects of the tankyrase inhibitor XAV-939 or of siRNA-mediated knockdown of tankyrases were evaluated in the mouse models of bleomycin-induced dermal fibrosis and in experimental fibrosis induced by adenoviral overexpression of a constitutively active TGF- $\beta$ receptor I (Ad-TBRI).

Results Inactivation of tankyrases prevented the activation of canonical Wnt signalling in experimental fibrosis and reduced the nuclear accumulation of $\beta$ catenin and the mRNA levels of the target gene c-myc. Treatment with XAV-939 or siRNA-mediated knockdown of tankyrases in the skin effectively reduced bleomycininduced dermal thickening, differentiation of resting fibroblasts into myofibroblasts and accumulation of collagen. Potent antifibrotic effects were also observed in Ad-TBRI driven skin fibrosis. Inhibition of tankyrases was not limited by local or systemic toxicity.

Conclusions Inactivation of tankyrases effectively abrogated the activation of canonical Wnt signalling and demonstrated potent antifibrotic effects in well-tolerated doses. Thus, tankyrases might be candidates for targeted therapies in fibrotic diseases.

\section{INTRODUCTION}

Canonical Wnt signalling belongs to the so-called morphogen pathways that are essential for development, but also tissue homeostasis. ${ }^{1} 2$ The central regulatory mechanism of canonical Wnt signalling is the proteolysis of $\beta$-catenin. In the absence of Wnt signals, cytosolic $\beta$-catenin is constitutively phosphorylated by the $\beta$-catenin destruction complex and targeted for proteasomal degradation. However, in the presence of Wnt proteins, the $\beta$-catenin destruction complex is inhibited, $\beta$-catenin is stabilised and translocates into the nucleus to initiate the transcription process of Wnt target genes. ${ }^{2}$

Aberrant activity of canonical Wnt signalling has been implicated in a variety of diseases ${ }^{3} 4$ and has recently emerged as a key pathway in systemic sclerosis (SSc). ${ }^{5-10}$ In SSc, the canonical Wnt pathway is activated as a result of a profound dysbalance between stimulatory and inhibitory mediators. While the ligands Wnt-1 and Wnt-10b are overexpressed, antagonists such as dickkopf-1, dickkopf -2 and Wnt-inhibitory factor are downregulated, in part in a TGF- $\beta$ dependent manner. ${ }^{6} 10$ Activation of canonical Wnt signalling stimulates resting fibroblasts to differentiate into myofibroblasts and release abundant amounts of extracellular matrix. Persistently activated canonical Wnt signalling induced by overexpression of either a stabilised mutant of $\beta$-catenin or Wnt-10b results in massive skin fibrosis. ${ }^{5} 6910$ Together, these findings indicate that canonical Wnt signalling may be an interesting target for antifibrotic therapies.

Targeted inhibition of canonical Wnt signalling has long been complicated by the lack of pathway components that are amenable to pharmacological inhibition. ${ }^{11}$ Only recently, tankyrases 1 and 2 (TNKS-1 and TNKS-2) were identified as promising targets. ${ }^{12}$ TNKS-1 and TNKS-2 stimulate the proteasomal degradation of axin, thereby inhibiting the degradation of $\beta$-catenin. Thus, pharmacologic inhibition of both tankyrases by XAV-939 (3,5,7,8-tetrahydro-2-[4-(trifluoromethyl)phenyl]$4 H$-thiopyrano $[4,3-d]$ pyrimidin-4-one) stabilised the $\beta$-catenin destruction complex, decreased the levels of $\beta$-catenin and selectively and efficiently inhibited canonical Wnt signalling. ${ }^{12}$

Considering the strong pro-fibrotic effects of canonical Wnt signalling, the availability of XAV-939 as a potent and selective inhibitor and the clinical need for antifibrotic therapies, we investigated inhibition of tankyrases as a novel targeted therapy for the treatment of fibrosis in preclinical models of scleroderma.

\section{MATERIAL AND METHODS}

\section{Experimental models of dermal fibrosis}

Bleomycin-induced dermal fibrosis was modelled as described. ${ }^{13} 14$ In a second model, fibrosis was induced by intradermal injections of replicationdeficient type 5 adenoviruses encoding for a constitutively active TGF- $\beta$ receptor type I mutant (Ad-TBRI). Injections of viruses encoding for LacZ (Ad-LacZ) served as controls. ${ }^{10} 15$

\section{Pharmacological inhibition of tankyrases}

XAV-939, a selective inhibitor of TNKS-1 and TNKS-2 (R\&D Systems GmbH, WiesbadenNordenstadt, Germany), was injected in doses of $2.5 \mathrm{mg} / \mathrm{kg}$ intraperitoneal four times a day (injection volume $100 \mu \mathrm{l}$ ). Control mice were sham 
injected with $100 \mu \mathrm{l}$ of $10 \% \mathrm{DMSO} / 90 \% 0.9 \% \mathrm{NaCl}$, the solvent of XAV-939.

\section{siRNA-mediated knockdown of TNKS-1 and TNKS-2}

Complexes of siRNA and atelocollagen (Koken, Tokyo, Japan) were prepared as described previously. ${ }^{16}{ }^{17}$ The following siRNA duplexes from Thermo Fisher Scientific (Bonn, Germany) were used: TNKS-1: 5'-GAUCCGAAUGCCAGAGAU AUU-3', antisense 5'-PUAUCUCUGGCAUUCGGAUCUU-3'; TNKS-2: 5'-GAGCUGAUGUUCAUGCUAAUU-3' and antisense 5'-PUUAGCAUGAACAUCAGCUCUU-3'. Non-targeting siRNA duplexes served as controls. Atelocollagen-siRNA complexes were injected intracutaneously once weekly into bleomycin challenged skin areas.

\section{Analysis of dermal fibrosis and adverse effects}

Dermal thickness, $\alpha$-smooth muscle actin and hydroxyproline content were analysed as described previously. ${ }^{5} 610 \quad 13-151819$ Mice were monitored daily for activity, weight loss, texture of the fur and diarrhoea.

\section{Triple staining for $\beta$-catenin, fibroblast-specific protein-1 and 4',6-diamidino-2-phenylindole (DAPI)}

Formalin-fixed, paraffin-embedded skin sections were stained for fibroblast-specific protein-1 (FSP-1) (Acris, Herford, Germany) and anti- $\beta$-catenin (Abcam, Cambridge, UK). Concentration-matched and species-specific immunoglobulins (Vector Laboratories, Burlingame, California, USA) served as control antibodies. After labelling with Alexa Fluor 594-tagged (red, $\beta$-catenin) and Alexa Fluor 488-tagged (green, FSP-1; both Invitrogen) secondary antibodies, sections were counterstained with DAPI. ${ }^{6} 10$

\section{Quantitative real time-PCR}

The levels of c-myc mRNA in total RNA from murine skin were quantified by SYBR green real time-PCR using the following primers: 5'-TGAAGGCTGGATTTCCTTTGGGCG-3', 5'-ATCGTCGTGGCTGTCGGGGT-3' ${ }^{10} 13$

\section{Statistics}

All data are presented as median with IOR, and differences between the groups were tested for their statistical significance by non-parametric Mann-Whitney $U$ test. A $p$ value of less than 0.05 was considered statistically significant; $p$ values are expressed as follows: $0.05>p>0.01$ as ${ }^{*} ; 0.01>p>0.001$ as ${ }^{* *}$; and $\mathrm{p}<0.001$ as ${ }^{* * *}$.

\section{RESULTS}

\section{Inactivation of tankyrases inhibits Wnt signalling} in experimental dermal fibrosis

Challenge with bleomycin as well as Ad-TBRI induced nuclear accumulation of $\beta$-catenin in dermal fibroblasts and upregulated the mRNA levels of the Wnt target gene c-myc as compared with non-fibrotic control mice (figure 1A,B). Pharmacological inhibition of tankyrases by XAV-939 completely abrogated the nuclear accumulation of $\beta$-catenin in fibroblasts (figure $1 \mathrm{~A}$ ) and also normalised the levels of c-myc in fibrotic skin (figure 1B). Similar results were obtained by siRNA-mediated knockdown (data not shown), demonstrating that targeting of tankyrases inhibits activation of canonical Wnt signalling in experimental fibrosis.

\section{Inactivation of tankyrases prevents bleomycin-induced dermal fibrosis}

Pharmacological inhibition of tankyrases by XAV-939 was well tolerated. No changes in the texture of the fur, activity or behaviour were observed in mice treated with XAV-939 compared with sham-treated controls during daily monitoring. We also did not observe signs of gastrointestinal toxicity such as weight loss or diarrhoea.

Inhibition of tankyrases exerted potent antifibrotic effects. Treatment of bleomycin challenged mice with XAV-939 reduced dermal thickening by $50 \%$ compared with sham-treated, bleomycin challenged mice $(p=0.0007)$ (median: 1.75 (IOR 1.60-2.00) for bleomycin and sham treatment vs median: 1.30 (IOR 1.10-1.41) for bleomycin + XAV-939) (figure 2A). The number of myofibroblasts and the hydroxyproline content were also significantly decreased in mice treated with XAV-939 (figure $2 \mathrm{~B}, \mathrm{C}$ ).

To exclude that the antifibrotic effects of XAV-939 are due to off-target effects, we targeted the expression of tankyrases in fibrotic skin by local injections of siRNAs against TNKS-1 and TNKS-2. siRNAs efficiently reduced the mRNA levels of TNKS- 1 and TNKS- 2 with minimal decreases of $64 \pm 10 \%$ at the end of the injection period $(p=0.04)$. siRNA-mediated knockdown of tankyrases reduced bleomycin-induced dermal thickening by $77 \pm 5 \%$ compared with mice injected with nontargeting siRNA ( $p=0.009$ ) (figure $2 \mathrm{D})$. Consistently, myofibroblast counts and the hydroxyproline content were also decreased by siRNAs treatment (figure $2 \mathrm{E}, \mathrm{F}$ ).

\section{Inhibition of tankyrases prevents Ad-TBRI-induced dermal fibrosis}

We have shown recently that TGF- $\beta$ signalling activates canonical Wnt signalling and that canonical Wnt signalling is a downstream mediator of the pro-fibrotic effects of TGF- $\beta$ signalling. ${ }^{10}$ Therefore, we examined whether inhibition of tankyrases prevents Ad-TBRI-induced fibrosis. Treatment with XAV-939 inhibited dermal thickening in Ad-TBRI-treated mice with reductions of $60 \%$ compared with sham-treated, Ad-TBRI mice (median: 1.99 (IOR 1.73-2.17) for sham-treated Ad-TBRI mice vs median: 1.43 (IOR 1.26-1.61) for XAV-939 treated Ad-TBRI mice) $(p=0.0009)$ (figure 3A). Myofibroblast counts and the hydroxyproline content were also significantly reduced by treatment with XAV-939 (figure 3B,C).

\section{DISCUSSION}

Canonical Wnt signalling has emerged as a central pathway for fibroblast activation in SSc and other fibrotic diseases. ${ }^{5-10}$ Pharmacological inhibition of canonical Wnt signalling, however, has long been hindered by the lack of molecular targets that are suitable for inhibition by small molecules. ${ }^{11} 20$ Here, we demonstrate that tankyrases might be promising candidates to target canonical Wnt signalling in fibrotic diseases. XAV-939, a selective small molecule inhibitor of tankyrases, ${ }^{12}$ effectively inhibited canonical Wnt signalling and exerted potent antifibrotic effects in preclinical models of fibrosis. Treatment with XAV-939 in pharmacologically relevant concentrations prevented both bleomycin-induced dermal fibrosis, a model for early, inflammatory stages of scleroderma, and Ad-TBRI-induced skin fibrosis, which serves as a model for non-inflammatory stages with endogenous activation of resident fibroblasts. In addition to the treatment with XAV-939, siRNA-mediated knockdown of TNKS-1 and TNKS-2 
A

$\mathrm{NaCl}$

sham

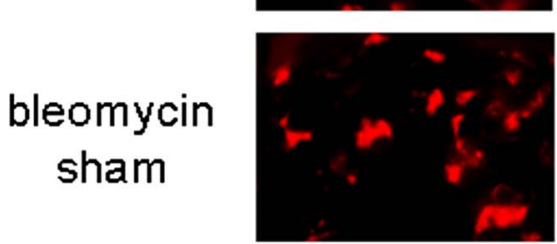

bleomycin

XAV-939

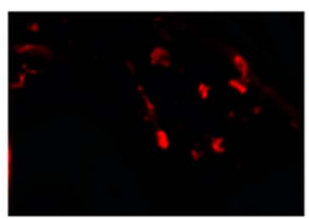

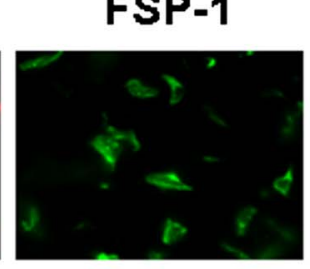

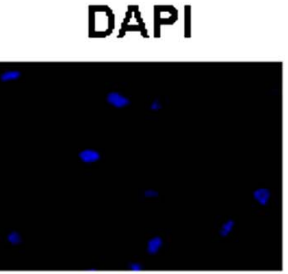

\section{overlay}
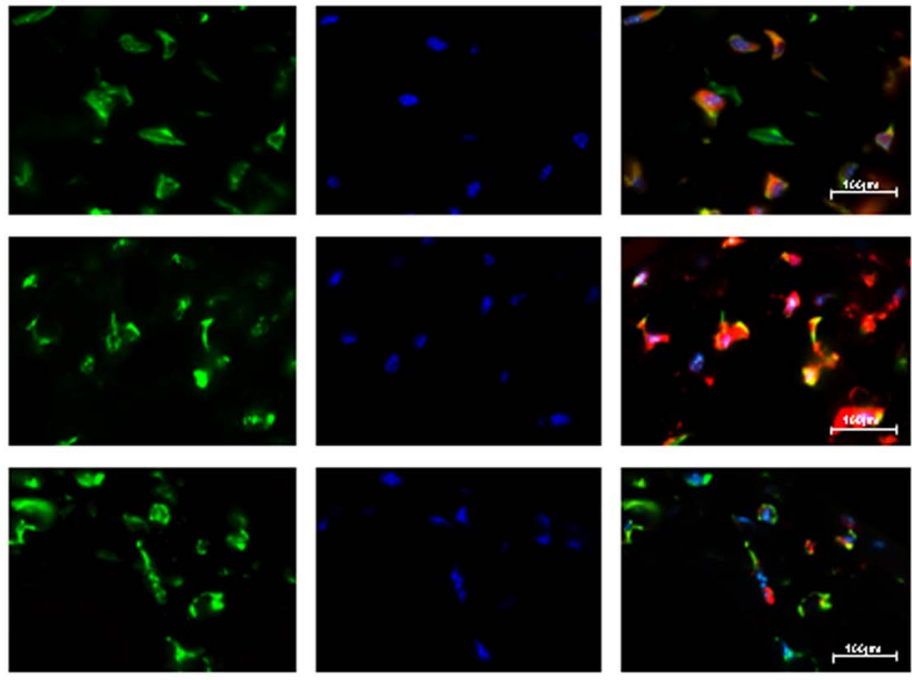

Ad-lacZ sham
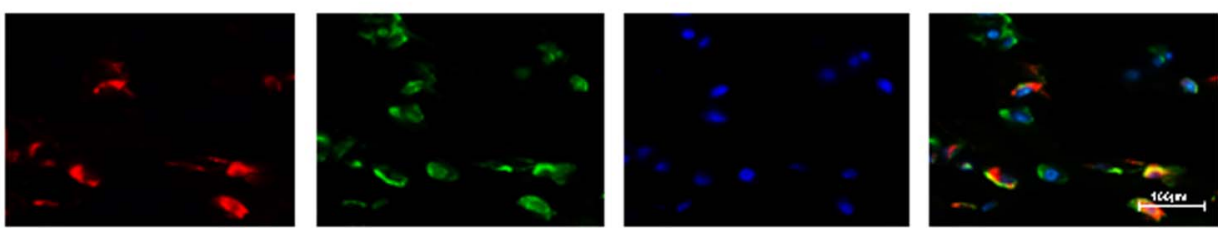

Ad-TBRI

sham
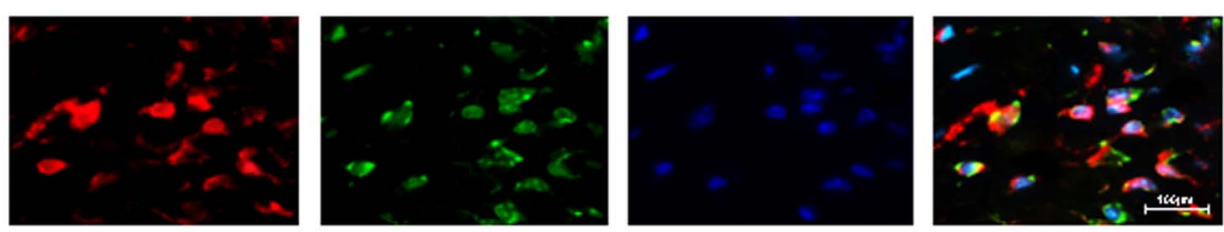

\section{Ad-TBRI \\ XAV939}
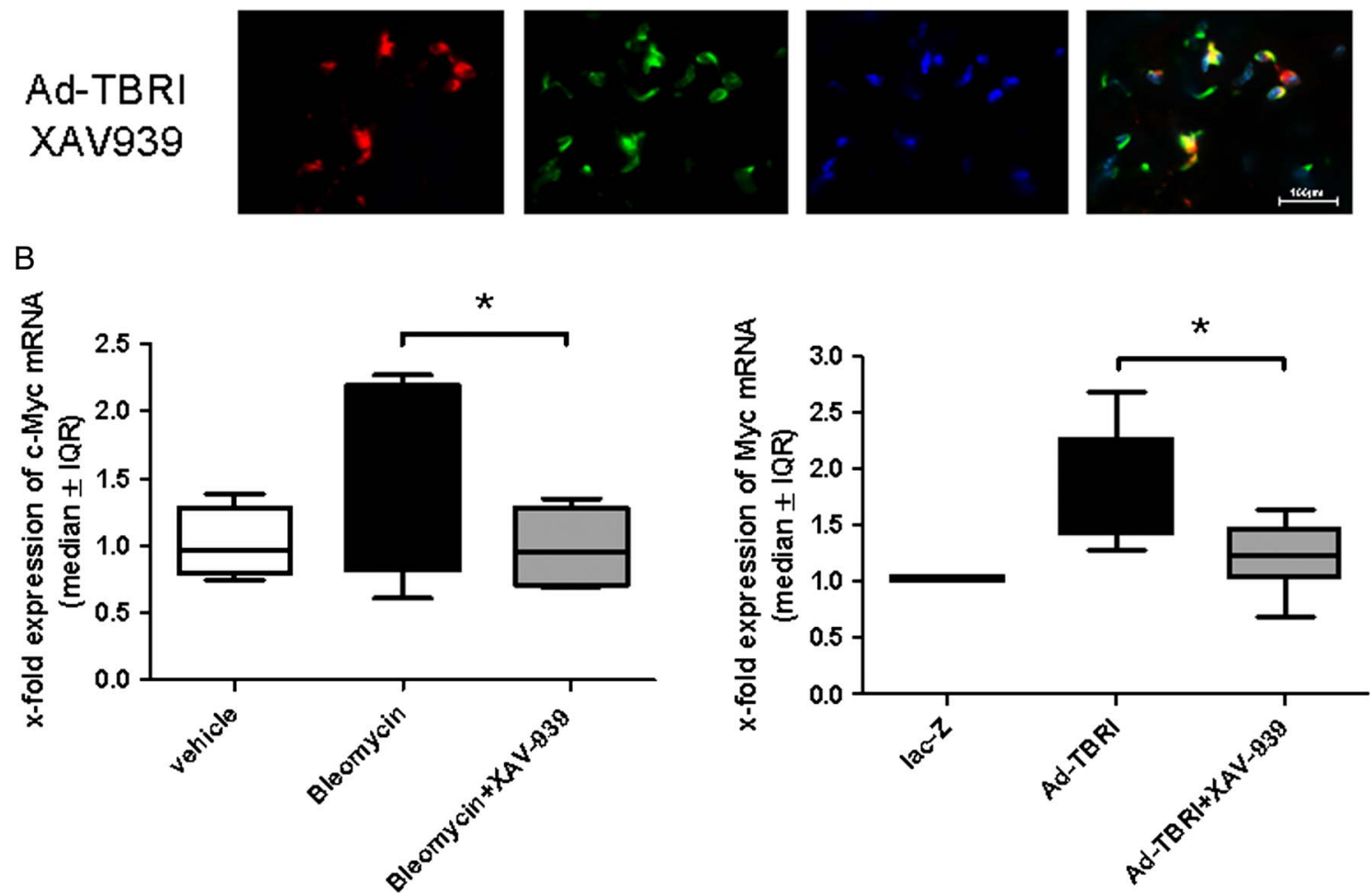

Figure 1 Inactivation of tankyrases reduces Wnt signalling in experimental dermal fibrosis. (A) Treatment with the tankyrase inhibitor XAV-939 inhibited the nuclear accumulation of $\beta$-catenin in fibroblast-specific protein-1 positive cells in bleomycin- and active TGF- $\beta$ receptor I (Ad-TBRI)-induced skin fibrosis. (B) Inhibition of tankyrases normalises the mRNA levels of c-myc in mice challenged with bleomycin or Ad-TBRI. $\mathrm{n}=5$ For each group. Access the article online to view this figure in colour. 


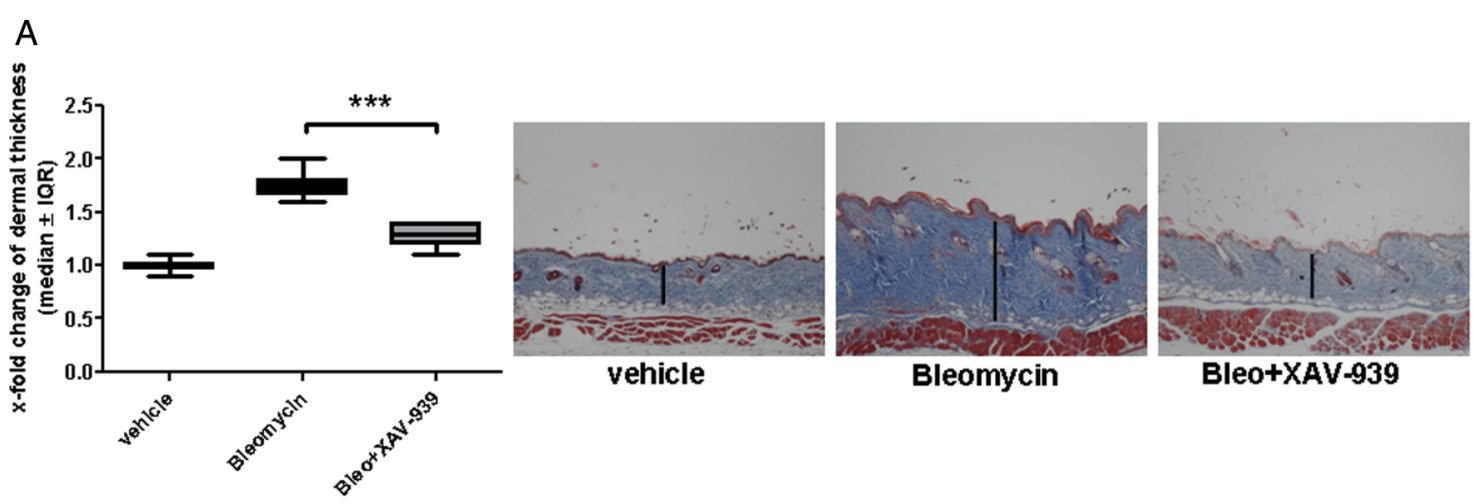

B
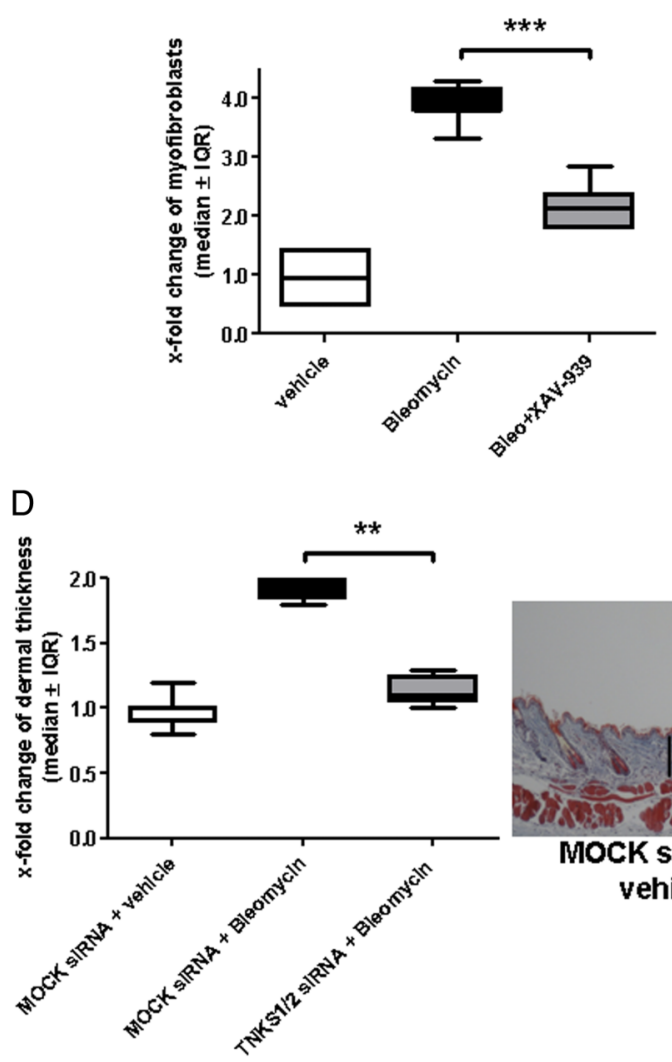

E

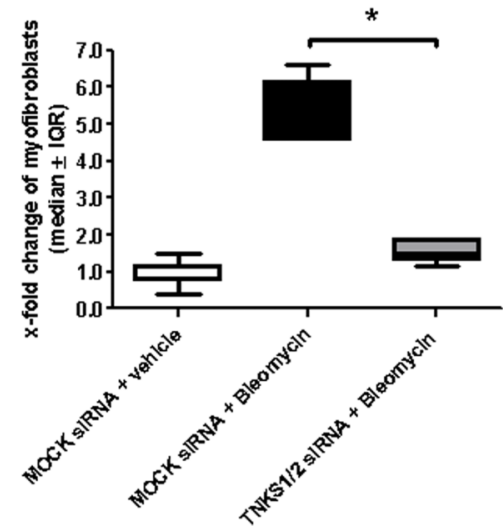

C

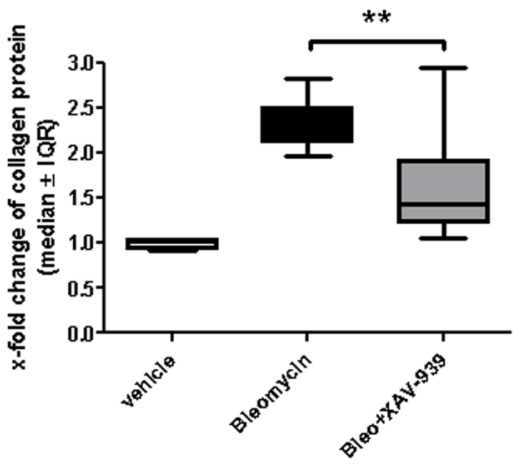

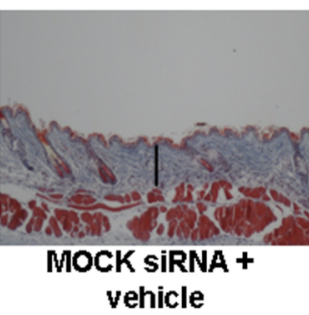

vehicle

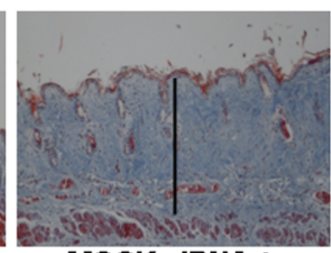

MOCK SIRNA + Bleomycin

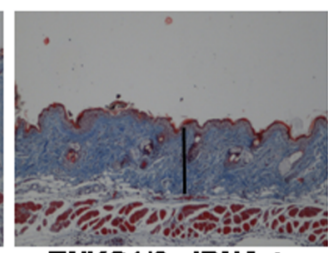

TNKS1/2 SIRNA +

Figure 2 Inactivation of tankyrases prevents bleomycin-induced dermal fibrosis. (A-C)Treatment with XAV-939 reduces dermal thickening (A), myofibroblast differentiation $(B)$ and hydroxyproline content $(C)$ in mice challenged with bleomycin. Representative trichrome-stained tissue sections are shown at 100 -fold magnification. $n=10$ For $\mathrm{NaCl}$ and bleomycin, $\mathrm{n}=8$ for XAV-939. (D-F) siRNA-mediated knockdown of tankyrases 1 and 2 (TNKS-1 and TNKS-2) prevented bleomycin-induced dermal thickening (D), differentiation of resting fibroblasts into myofibroblasts (E) and reduced the hydroxyproline content (F). Representative sections are shown at 100 -fold magnification. $n=5$ For each group. Access the article online to view this figure in colour. 

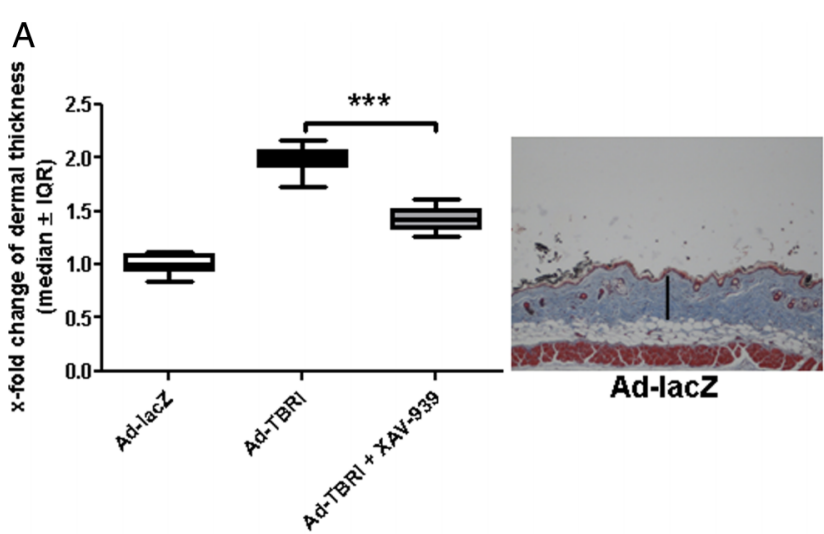

Ad-lacZ

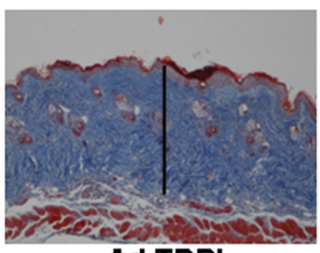

Ad-TBRI

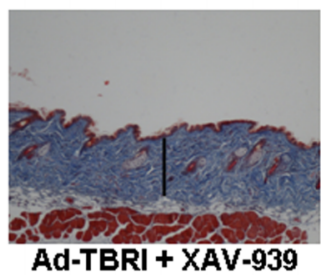

Ad-TBRI + XAV-939
B

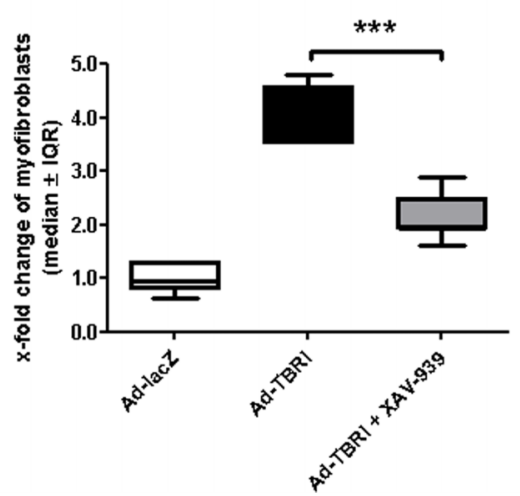

C

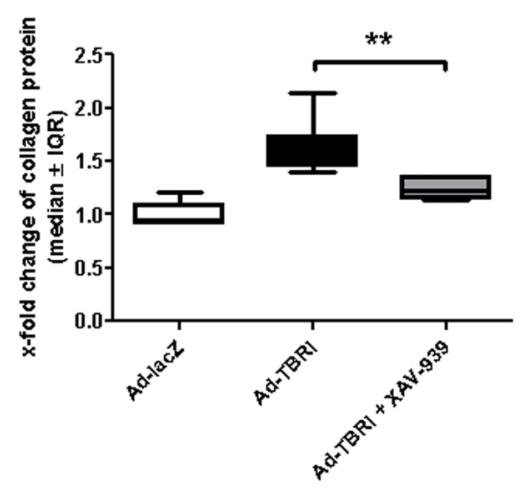

Figure 3 Inhibition of tankyrases prevents active TGF- $\beta$ receptor I (Ad-TBRI)-induced fibrosis. Treatment with XAV-939 prevents dermal thickening (A), myofibroblast differentiation (B) and hydroxyproline content (C) in mice infected with Ad-TBRI. Representative trichrome-stained tissue sections are shown at 100 -fold magnification. $n=5$ For each group. Access the article online to view this figure in colour.

prevented experimental fibrosis, confirming that the antifibrotic effects of XAV-939 were on target.

Besides lack of molecular targets, toxicity has been a concern when inhibiting canonical Wnt signalling. ${ }^{11} 20$ In particular, impaired stem cell renewal with reduced regeneration of tissues with high cell turnover such as the gastrointestinal tract or the hair follicles has been discussed as dose limiting complications. Nevertheless, treatment of mice with XAV-939 in antifibrotic doses for up to 8 weeks was well tolerated. We did not observe diarrhoea or weight loss as clinical signs of gastrointestinal toxicity. Furthermore, texture of the fur and the macroscopic architecture of the hair bulb appeared to be normal upon treatment with XAV-939.

These data present first evidence that inactivation of tankyrases: (i) effectively inhibits canonical Wnt signalling, (ii) exerts potent antifibrotic effects in preclinical models representing different stages of scleroderma and (iii) is not limited by toxicity in mice. Tankyrases may thus be an interesting novel candidate for targeted therapies in fibrotic disease. Nevertheless, further studies are needed to investigate the antifibrotic effects of tankyrase inhibitors in other organs and on vascular manifestations, to analyse regression of fibrosis, and to exclude toxicity in other species and upon long-term treatment.

Acknowledgements We thank Anna-Maria Herrmann, Madeleine Demleitner, Verena Wäsch and Stefan Fritz for excellent technical assistance.

Contributors Design of the study: $A D, J H W D$. Acquisition of data: $A D, L D, J H, C D$, $A W, N-Y L, C B$. Interpretation of data: $A D, L D, O D, G S, J H W D$. Manuscript preparation: $A D, J H W D$.

Funding Grants DI 1537/1-1, DI 1537/2-1, DI 1537/4-1, DI 1537/5-1, AK 144/1-1, WE 4275/3-1 and SCHE 1583/7-1 of the Deutsche Forschungsgesellschaft, grants
A20 and A40 of the IZKF in Erlangen, the ELAN-Program of the University of Erlangen-Nuremberg and the Career Support Award of Medicine of the Ernst Jung Foundation and Dr Robert-Pfleger-Stiftung.

Competing interests PD Dr 0 Distler had consultancy relationship and/or has received research funding from Actelion, Bayer, Pfizer, Ergonex, BMS, Sanofi-Aventis, Sinoxa, United BioSource Corporation, medac, Biovitrium, Boehringer Ingelheim Pharma, Novartis, 4 D Science and Active Biotec in the area of potential treatments of scleroderma and its complications. JHW Distler has consultancy relationships and/ or has received research funding from Actelion, Pfizer, Ergonex, BMS, Celgene, Bayer Pharma, Boehringer Ingelheim, JB Therapeutics, Sanofi-Aventis, Novartis, Array Biopharma and Active Biotec in the area of potential treatments of scleroderma and is stock owner of $4 \mathrm{D}$ Science $\mathrm{GmbH}$.

Patient consent Obtained.

Ethics approval This study was conducted with the approval of the ethical committees of the University of Erlangen-Nuremberg, Germany.

Provenance and peer review Not commissioned; externally peer reviewed.

Data sharing statement Additional information is available from the corresponding author upon request.

\section{REFERENCES}

1. Logan CY, Nusse R. The Wnt signaling pathway in development and disease. Annu Rev Cell Dev Biol 2004;20:781-810.

2. van Amerongen $\mathbf{R}$, Nusse R. Towards an integrated view of Wnt signaling in development. Development 2009;136:3205-14.

3. MacDonald BT, Tamai K, He X. Wnt/beta-catenin signaling: components, mechanisms, and diseases. Dev Cell 2009;17:9-26.

4. Pinzone JJ, Hall BM, Thudi NK, et al. The role of Dickkopf-1 in bone development, homeostasis, and disease. Blood 2009;113:517-25.

5. Bergmann C, Akhmetshina A, Dees $C$, et al. Inhibition of glycogen synthase kinase 3beta induces dermal fibrosis by activation of the canonical Wnt pathway. Ann Rheum Dis 2011;70:2191-8.

6. Beyer C, Schramm A, Akhmetshina A, et al. beta-catenin is a central mediator of pro-fibrotic Wnt signaling in systemic sclerosis. Ann Rheum Dis 2012;71:761-7. 


\section{Basic and translational research}

7. Lam AP, Flozak AS, Russell $\mathrm{S}$, et al. Nuclear beta-catenin is increased in systemic sclerosis pulmonary fibrosis and promotes lung fibroblast migration and proliferation. Am J Respir Cell Mol Biol 2011;45:915-22.

8. Wei J, Fang F, Lam AP, et al. Wnt/beta-catenin signaling is hyperactivated in systemic sclerosis and induces Smad-dependent fibrotic responses in mesenchymal cells. Arthritis Rheum 2012;64:2734-45.

9. Wei J, Melichian D, Komura K, et al. Canonical Wnt signaling induces skin fibrosis and subcutaneous lipoatrophy: a novel mouse model for scleroderma? Arthritis Rheum 2011;63:1707-17.

10. Akhmetshina A, Palumbo K, Dees C, et al. Activation of canonical Wnt signalling is required for TGF-beta-mediated fibrosis. Nat Commun 2012;3:735.

11. Barker $\mathbf{N}$, Clevers H. Mining the Wnt pathway for cancer therapeutics. Nat Rev Drug Discov 2006;5:997-1014.

12. Huang SM, Mishina YM, Liu S, et al. Tankyrase inhibition stabilizes axin and antagonizes Wnt signalling. Nature 2009;461:614-20.

13. Dees C, Akhmetshina A, Zerr P, et al. Platelet-derived serotonin links vascular disease and tissue fibrosis. J Exp Med 2011;208:961-72.
14. Dees C, Zerr P, Tomcik M, et al. Inhibition of Notch signaling prevents experimental fibrosis and induces regression of established fibrosis. Arthritis Rheum 2011;63:1396-404.

15. Avouac J, Palumbo K, Tomcik M, et al. Inhibition of activator protein 1 signaling abrogates transforming growth factor beta-mediated activation of fibroblasts and prevents experimental fibrosis. Arthritis Rheum 2012;64:1642-52.

16. Minakuchi $\mathbf{Y}$, Takeshita F, Kosaka N, et al. Atelocollagen-mediated synthetic small interfering RNA delivery for effective gene silencing in vitro and in vivo. Nucleic Acids Res 2004;32:e109.

17. Horn A, Kireva T, Palumbo-Zerr K, et al. Inhibition of hedgehog signalling prevents experimental fibrosis and induces regression of established fibrosis. Ann Rheum Dis 2012; 71:785-9.

18. Beyer C, Reich N, Schindler SC, et al. Stimulation of soluble guanylate cyclase reduces experimental dermal fibrosis. Ann Rheum Dis 2012;71:1019-26.

19. Zerr P, Palumbo-Zerr K, Distler A, et al. Inhibition of hedgehog signaling for the treatment of murine sclerodermatous chronic graft-versus-host disease. Blood 2012; 120:2909-17.

20. Yao H, Ashihara E, Maekawa T. Targeting the Wnt/beta-catenin signaling pathway in human cancers. Expert Opin Ther Targets 2011;15:873-87. 


\section{Inactivation of tankyrases reduces} experimental fibrosis by inhibiting canonical Wnt signalling

Alfiya Distler, Lisa Deloch, Jingang Huang, Clara Dees, Neng-Yu Lin, Katrin Palumbo-Zerr, Christian Beyer, Alexander Weidemann, Oliver Distler, Georg Schett and Jörg H W Distler

Ann Rheum Dis 2013 72: 1575-1580 originally published online November 12, 2012

doi: 10.1136/annrheumdis-2012-202275

Updated information and services can be found at:

http://ard.bmj.com/content/72/9/1575

\section{These include:}

References This article cites 20 articles, 9 of which you can access for free at: http://ard.bmj.com/content/72/9/1575\#BIBL
Email alerting service
Receive free email alerts when new articles cite this article. Sign up in the box at the top right corner of the online article.

Topic Articles on similar topics can be found in the following collections

Collections

Connective tissue disease (4234)

Genetics (964)

Occupational and environmental medicine (29)

\section{Notes}

To request permissions go to:

http://group.bmj.com/group/rights-licensing/permissions

To order reprints go to:

http://journals.bmj.com/cgi/reprintform

To subscribe to BMJ go to:

http://group.bmj.com/subscribe/ 